\title{
ON THE EXISTENCE OF IMMERSIONS AND SUBMERSIONS
}

\author{
BY \\ EMERY THOMAS $\left({ }^{1}\right)$
}

1. Introduction. Let $M$ and $N$ be manifolds (always assumed to be smooth, connected, and without boundary) and let $f: M \rightarrow N$ be a smooth map. If at each point of $M$ the Jacobian matrix of $f$ has maximal rank, we call $f$ a map of maximal rank. (If $\operatorname{dim} M<\operatorname{dim} N$, then $f$ is an immersion, while if $\operatorname{dim} M>\operatorname{dim} N, f$ is a submersion.)

QUESTION. Which homotopy classes of continuous maps $M \rightarrow N$ contain a smooth map of maximal rank?

This question has been reduced to a question purely in homotopy theory by M. Hirsch (for immersions) and Phillips (for submersions). (See [7], [17].) Their results are as follows.

We will use the following notation. For any vector bundle $\xi$ over a complex $X$ we let $(\xi)$ denote the stable equivalence class determined by $\xi$. We will say that a stable bundle $(\xi)$ has geometric dimension $\leqq n$ (for some positive integer $n$ ) if there is an $n$-plane bundle over $X$ which is stably isomorphic to $\xi$. For a smooth manifold $V$ we let $\tau_{V}$ denote the tangent bundle and $\nu_{V}$ the stable normal bundle; i.e. $\nu_{V}=-\left(\tau_{V}\right)$.

THEOREM OF HiRSCH. Let $f: M \rightarrow N$ be a continuous map between manifolds, where $\operatorname{dim} M<\operatorname{dim} N$. Then $f$ is homotopic to an immersion if, and only if, the stable bundle

$$
f^{*}\left(\tau_{N}\right)+\nu_{M}
$$

has geometric dimension $\leqq \operatorname{dim} N-\operatorname{dim} M$.

A dual result holds for submersions.

TheORem of Phillips. Let $M$ be an open manifold and $f: M \rightarrow N$ a continuous map, where $\operatorname{dim} M>\operatorname{dim} N$. Then $f$ is homotopic to a submersion if, and only if, the stable bundle

$$
\left(\tau_{M}\right)+f^{*} \nu_{N}
$$

has geometric dimension $\leqq \operatorname{dim} M-\operatorname{dim} N$.

Received by the editors March 2, 1967.

(1) Research supported by the National Science Foundation. The author is a Professor in the Miller Institute for Basic Research (Berkeley). 
We need here to remark that since $M$ is open, the bundle $\tau_{M}$ is stable over $M$. (Because $M$ has the homotopy type of an $(m-1)$-complex, $m=\operatorname{dim} M$. See $[8, \S 3.2]$.)

For a simple application of these theorems, suppose that $M$ and $N$ are $\pi$-manifolds (i.e. each has stably trivial tangent bundle) and that $\operatorname{dim} M \neq \operatorname{dim} N$. Since each also has stably trivial normal bundle, it follows by the above theorems that every continuous map $M \rightarrow N$ is homotopic to a smooth map of maximal rank.

In the following two sections we use the theorems of Hirsch and Phillips to study more general manifolds $M$ and $N$, using in part results from [20] and [21].

2. Immersion of manifolds. We will use the following notation. $M^{m}$ and $N^{n}$ will denote smooth connected manifolds with respective dimensions $m$ and $n$, $m<n$. We define the codimension of a continuous map $M \rightarrow N$ to be the positive integer $n-m$. By the basic theorem of Whitney [23] every map of codimension $m$ (i.e. $n=2 m$ ) is homotopic to an immersion, and so we consider here the case $n<2 m$.

For any bundle $\xi$ over a complex $X$ we let $w_{i} \xi \in H^{i}\left(X ; Z_{2}\right)$ denote the $i$ th StiefelWhitney class of $\xi, i \geqq 0$. For a manifold $V$, we set

$$
w_{i}(V)=w_{i}\left(\tau_{V}\right), \quad \bar{w}_{i}(V)=w_{i}\left(v_{V}\right) .
$$

Suppose now that $M$ and $N$ are manifolds and $f: M \rightarrow N$ a continuous map. Set

$$
\nu_{f}=f^{*}\left(\tau_{N}\right)+\nu_{M}
$$

We say that $f$ is orientable if

$$
f^{*} w_{1}(N)=w_{1}(M)
$$

i.e. the stable bundle $v_{f}$ is orientable.

By Hirsch (see $\$ 1$ ), it follows that if $f$ is homotopic to an immersion then

$$
\begin{aligned}
w_{i}\left(v_{f}\right) & =0, & & i>n-m, \\
\delta w_{n-m}\left(v_{f}\right) & =0, & & n-m \text { even, } f \text { orientable. }
\end{aligned}
$$

(Here $\delta$ denotes the Bockstein coboundary associated with the exact sequence $Z \rightarrow Z \rightarrow Z_{2}$.) Thus, in what follows we will be mainly concerned with sufficient conditions for $f$ to be homotopic to an immersion.

Codimension $f=m-1, m \geqq 4$.

Theorem 2.1. Let $M^{m}$ and $N^{2 m-1}$ be manifolds, $m \geqq 4$, and let $f: M \rightarrow N$ be a continuous map. If $m$ is odd, assume that $f$ is orientable. Then $f$ is homotopic to an immersion if, and only if,

$$
w_{m}\left(v_{f}\right)=0, \quad m \text { even, } \quad \delta w_{m-1}\left(v_{f}\right)=0, \quad m \text { odd } .
$$

The proof of the theorem follows at once from classical obstruction theory [18], as will be shown in $\S 5$. (In the case $m$ odd we can omit the hypothesis that $f$ is orientable if we use local coefficients.) 
If $M$ and $N$ are orientable manifolds, then every map $f: M \rightarrow N$ is orientable. Since $H^{m}(M ; Z) \approx Z$, we then obtain from 2.1

Corollary 2.2. Let $M^{2 q+1}$ and $N^{4 q+1}$ be orientable manifolds, $q \geqq 2$. Then every map $f: M \rightarrow N$ is homotopic to an immersion.

Codimension $f=m-2, m \geqq 5$.

TheOREM 2.3. (a) Let $M^{4 q+1}$ and $N^{8 q}$ be manifolds, $q \geqq 1$, and $f: M \rightarrow N$ a continuous map. Then $f$ is homotopic to an immersion if $w_{4 q}\left(\nu_{f}\right)=0$.

(b) Let $M^{4 q+2}$ and $N^{8 q+2}$ be manifolds, $q \geqq 1$, and let $f: M \rightarrow N$ be an orientable map. Suppose that

$$
\delta w_{4 q}\left(v_{f}\right)=0 \text { and } w_{4 q+2}\left(v_{f}\right)=0 .
$$

If $M$ is closed, suppose also that $M$ is orientable and that

$$
f^{*} w_{2}(N)=0, \quad w_{4 q}\left(v_{f}\right) \cdot w_{2}(M)=0 .
$$

Then $f$ is homotopic to an immersion.

(c) Let $M^{4 q+3}$ and $N^{8 q+4}$ be manifolds, $q \geqq 1$, and let $f: M \rightarrow N$ be an orientable map. Suppose that $w_{4 q+2}\left(v_{f}\right)=0$. If $M$ is open or if $M$ is closed, orientable, and either $f^{*} w_{2}(N) \neq 0$ or

$$
f^{*} w_{2}(N)=0 \text { and } w_{4 q+1}\left(v_{f}\right) \cdot w_{2}(M)=0,
$$

then $f$ is homotopic to an immersion.

The proof uses the results of [20], and will be given in $\$ 5$.

We say that an orientable manifold $M$ is a spin manifold if $w_{2}(M)=0$; we say that an orientable map $f: M \rightarrow N$ is a spin map if $f^{*} w_{2} N=w_{2} M$.

Codimension $f=m-3, m \geqq 5$.

THEOREM 2.4. Let $f: M^{m} \rightarrow N^{2 m-3}$ be an orientable map, with $m \geqq 5$ and $m \not \equiv 0 \bmod 4$. If $m \equiv 1 \bmod 4$, assume that $H^{m-1}\left(M ; Z_{2}\right)=0$. If $m \equiv 2 \bmod 4$, assume that either $M$ is open or that $M$ is a closed spin manifold and $f$ is a spin map. If $m \equiv 3 \bmod 4$, assume that $M$ is a closed spin manifold and $f$ is a spin map. Then $f$ is homotopic to an immersion if

$$
\begin{array}{rlrl}
\delta w_{m-3}\left(v_{f}\right) & =0, & & m \equiv 1 \\
w_{m-2}\left(v_{f}\right) & =0, & & \bmod 4 \\
\delta w_{m-3}\left(v_{f}\right) & =0, & w_{m-1}\left(v_{f}\right)=0, & m \equiv 3 \bmod 4 \\
& & m
\end{array}
$$

The proof will be given in $\S 5$.

Codimension $f=m-4, m \geqq 11$.

THEOREM 2.5. Let $M^{8 q+3}$ and $N^{16 q+2}$ be manifolds, $q \geqq 1$, and let $f: M \rightarrow N$ be a spin map. Suppose that $M$ is a closed spin manifold. If $w_{8 q}\left(v_{f}\right)=0$, then $f$ is homotopic to an immersion. 
The proof will be given in $\S 5$.

RemarK. If one takes the manifold $N$ to be $R^{n}$, then $\nu_{f}=v_{M}$ and one can obtain stronger results than those given in 2.1-2.4 by using [14]. Note, for example, [6], [11] and [21].

3. Submersion of manifolds. In this section we assume that $M^{m}$ and $N^{n}$ are smooth connected manifolds with $m>n$. Moreover, throughout the section we assume that $M$ is open. Suppose that $n=1$, i.e. $N=R^{1}$ or $S^{1}$. Then, as observed by Phillips [17], every map $M^{m} \rightarrow N^{1}$ is homotopic to a submersion (since $M$ has the homotopy type of an $(m-1)$-complex). We consider here the case $n=2$. For a map $f: M \rightarrow N$ set $\sigma_{f}=\left(\tau_{M}\right)+f^{*} \nu_{N}$. We will prove

THEOREM 3.1. Let $f: M^{m} \rightarrow N^{2}$ be a continuous map, $m \geqq 5$, where $M$ is open. If $m$ is even assume that $f$ is orientable. Then $f$ is homotopic to a submersion if, and only if,

$$
w_{m-1}\left(\sigma_{f}\right)=0, \quad m \text { odd }, \quad \delta w_{m-2}\left(\sigma_{f}\right)=0, \quad m \text { even } .
$$

Suppose that $N$ is a closed orientable surface. Then the stable normal bundle of $N$ is trivial, and so $w_{i}\left(\sigma_{f}\right)=w_{i}(M), i \geqq 0$.

On the other hand suppose that $M=M^{\prime}-\partial M^{\prime}$, where $M^{\prime}$ is a compact orientable manifold with nonempty boundary $\partial M^{\prime}$. It follows from results of $\mathrm{Wu}$ and Massey [24], [12], [13], that

$$
w_{m-1}(M)=0, \quad \text { if } m \equiv 3 \quad \bmod 4, \quad \delta w_{m-2}(M)=0, \quad \text { if } m \text { even } .
$$

(See [5, §2].) Thus from 3.1 we obtain

COROLlaRY 3.2. Let $M^{\prime}$ be a compact orientable m-manifold with nonempty boundary $\partial M^{\prime}$, and let $N$ be a closed orientable surface. Let $M$ denote the open manifold $M^{\prime}-\partial M^{\prime}$. If $m \geqq 5$ and $m \not \equiv 1 \bmod 4$, then every map $M \rightarrow N$ is homotopic to a submersion.

(Note [3] for conditions on an open manifold that it be expressible as $M^{\prime}-\partial M^{\prime}$.)

Our results on submersions are much less extensive than the results in $\S 2$ on immersions. If $f: M^{m} \rightarrow N^{n}$, with $n>2$, then one can still apply the results of [20], [21] to obtain conditions for $\sigma_{f}$ to have codimension $\leqq m-n$. However, the results in general will be expressed in terms of higher order cohomology operations.

4. Examples. Let $M^{m}$ and $N^{n}$ be manifolds, $m \neq n$. The problem of determining the set of maps from $M$ to $N$ of maximal rank falls into two parts: First, determine the homotopy classes of maps from $M$ to $N,[M, N]$; and second, for each homotopy class of maps, determine whether it contains a map of maximal rank. If $M$ and $N$ fit the hypotheses of one of the theorems in $\S 2$ or $\S 3$, and if $f: M \rightarrow N$, then the second step above consists simply in computing the characteristic classes 
$w_{k}\left(v_{f}\right)$, if $m<n, w_{k}\left(\sigma_{f}\right)$, if $m>n$. By the Whitney duality formula, these classes are given as follows:

$$
w_{k}\left(\nu_{f}\right)=\sum_{i+j=k} \bar{w}_{i}(M) \cup f^{*} w_{j}(N), \quad w_{k}\left(\sigma_{f}\right)=\sum_{i+j=k} w_{i}(M) \cup f^{*} \bar{w}_{j}(N) .
$$

For an illustration we take $N$ to be the real projective space $R P^{n}$ (of $\operatorname{dim} n$ ) and the complex projective space $C P^{n}$ (of $\operatorname{dim} 2 n$ ).

EXAmple A. $N=R P^{n}, n>1$. Since $R P^{n}$ is the $n$-skeleton of the EilenbergMacLane space $K\left(Z_{2}, 1\right)$, it follows that if $X$ is a complex of $\operatorname{dim}<n$, then $\left[X, R P^{n}\right]$ $=H^{1}\left(X ; Z_{2}\right)$. The correspondence here is given by $[f] \rightarrow f^{*} x$, where $x$ generates $H^{1}\left(R P^{n} ; Z_{2}\right)$. Since $w\left(R P^{n}\right)=(1+x)^{n+1}$, we have

$$
w_{k}\left(v_{f}\right)=\sum_{i+j=k}\left(\begin{array}{c}
n+1 \\
i
\end{array}\right) u^{i} \cup \bar{w}_{j}(M),
$$

where $f: M^{m} \rightarrow R P^{n}, m<n$, and $u=f^{*} x$. The results of $\S 2$ can now be used to determine the immersions of $M^{m}$ in $R P^{n}$, for appropriate dimensions $m$ and $n$. (The difficulty in studying submersions is that in general we do not know how to determine the set $\left[M^{m}, R P^{n}\right]$, when $m>n$.)

EXAmPle B. $N=C P^{n}, n \geqq 1$. Now $C P^{n}$ is the $(2 n+1)$-skeleton of the EilenbergMacLane space $K(Z, 2)$, and so if a complex $X$ has dimension $\leqq 2 n$, then $\left[X, C P^{n}\right]$ $=H^{2}(X ; Z)$, the correspondence being given by $[f] \rightarrow f^{*} y$, where $y$ generates $H^{2}\left(C P^{n} ; Z\right)$. Let $M^{m}$ be a manifold and $f: M^{m} \rightarrow C P^{n}$ a map, $m \leqq 2 n$. Since $w\left(C P^{n}\right)=(1+y)^{n+1} \bmod 2$, we have

$$
w_{2 k}\left(\nu_{f}\right)=\sum_{i+j=k}\left(\begin{array}{c}
n+1 \\
i
\end{array}\right) v^{i} \cup \bar{w}_{2 j}(M), \quad \delta w_{2 k}\left(v_{f}\right)=\sum_{i+j=k}\left(\begin{array}{c}
n+1 \\
i
\end{array}\right) v^{i} \cup \delta \bar{w}_{2 j}(M),
$$

where $v=f^{*} y$. The results of $\S 2$ can now be used to determine the immersions of $M^{m}$ in $C P^{n}$ for appropriate $m$ and $n$. Take $M$ to be $C P^{q}$, for example. Since $H^{2}\left(C P^{q} ; Z\right) \approx Z$, we have $\left[C P^{q}, C P^{n}\right]=Z, q \leqq n$, and so each homotopy class of map $f: C P^{q} \rightarrow C P^{n}$ is characterized by an integer, called the degree of the map. (See Feder [4].) By 2.3(b) one can show:

(4.1) Let $q$ be a positive integer. Then for each integer $d$ there is an immersion of $C P^{2 q+1}$ in $C P^{4 q+1}$ of degree $d$.

REMARK. (4.1) suggests the following general problem. Let $q$ and $n$ be integers, $0<q<n$. Determine the integers $d$ for which there is an immersion of $C P^{q}$ in $C P^{n}$ of degree $d$. By Whitney [23], if $n \geqq 2 q$ all integers $d$ can occur. By Feder [4], if $n \leqq[3 q / 2]-1$, only $d= \pm 1$ can occur. (In [22] we show that for $q=2, n=3$, only $d= \pm 1$ can occur, while if $q=3, n=4$, then $d$ can occur if, and only if, there is an integer $e$ such that $5 d^{2}=e^{2}+4$. Note also [4, Theorem 8.3].)

5. Proofs of theorems. For a topological group $G$ let $B G$ denote the classifying space for $G$ constructed by Milnor [15]. Let $O(n), n \geqq 1$, denote the orthogonal group of rank $n$, and let $O$ denote the stable orthogonal group [2]. If $X$ is a complex 
then a stable vector bundle over $X$ can be regarded as a map $X \rightarrow B O$. Now the natural inclusion $O(n) \subset O$ induces a map $p_{n}: B O(n) \rightarrow B O$, and a stable bundle $\xi$ over $X$ has geometric dimension $\leqq n$ if, and only if, there is a map $\eta: X \rightarrow B O(n)$ such $p_{n} \circ \eta=\xi$. Up to homotopy type the map $p_{n}$ can be regarded as a fiber map [1], with fiber $V_{n}=O / O(n)$.

By Stiefel (see [18]), $V_{n}$ is $(n-1)$-connected and (for $n \geqq 3$ ),

$$
\begin{aligned}
\pi_{n}\left(V_{n}\right) & =Z, & & n \text { even }, \\
& =Z_{2}, & & n \text { odd. }
\end{aligned}
$$

Thus by standard obstruction theory (e.g. see [18], [10], or [19]), if $X$ has $\operatorname{dim} \leqq n+1$ then a stable bundle $\xi$ over $X$ has geometric $\operatorname{dim} \leqq n$ if, and only if,

$$
w_{n+1}(\xi)=0, \quad n \text { odd, } \quad \delta w_{n}(\xi)=0, \quad n \text { even, }
$$

assuming that $\xi$ is orientable in the case $n$ even. This proves Theorem 2.1. Furthermore, $(*)$ proves $2.3(\mathrm{a})$ (since $\pi_{4 q}\left(V_{4 q-1}\right)=0$, see [16]) and also proves 2.4 in the case $m \equiv 1 \bmod 4$ (since $\pi_{4 q+1}\left(V_{4 q-2}\right)=0$, [16]). Finally, since an open $m$-manifold has the homotopy type of an $(m-1)$-complex, $(*)$ also proves 3.1 , and $2.3-2.4$ in the cases $M$ is open.

To prove the remaining theorems in $\$ 2$ (assuming now that $M$ is a closed manifold) we need some results from [20], and [21]. In [20] we do not deal with stable bundles, and so we will need the following relationship between $n$-plane bundles and stable bundles.

Lemma 5.1. Let $X$ be a complex of $\operatorname{dim} n$ and let $\xi$ be an oriented stable vector bundle over $X$ such that $w_{n}(\xi)=0$. Then there is an oriented n-plane bundle $\eta$ over $X$ such that $\eta$ is stably equivalent to $\xi$ and $\chi(\eta)=0$ (where $\chi(\eta)$ denotes the Euler class of $\eta$ ). Moreover, $\xi$ has geometric dimension $\leqq k$ (where $k<n)$ if, and only if, $\eta$ has $n-k$ linearly independent cross-sections.

The proof is standard and is left to the reader.

Proof of 2.3(b). Let $\eta$ be an $n$-plane bundle over $M$ corresponding to the stable bundle $\nu_{f}$. Thus by 5.1 and by the hypotheses of 2.3(b),

$$
w_{2}(\eta)=w_{2}(M), \quad w_{4 q}(\eta) \cdot w_{2}(M)=0, \quad \delta w_{4 q}(\eta)=0, \quad \chi(\eta)=0,
$$

and so by Theorem 7.3 of [20], $\eta$ has 2 linearly independent cross sections. Thus, by $5.1, \nu_{f}$ has geometric dimension $\leqq 4 q$ and so by Hirsch, $f$ is homotopic to an immersion.

Before proving $2.3(\mathrm{c})$ we need a preliminary result. Let $\xi$ be a vector bundle (stable or otherwise) over a complex $X$. Define a homomorphism

$$
\alpha_{\xi}: H^{i}\left(X ; Z_{2}\right) \rightarrow H^{i+2}\left(X ; Z_{2}\right), \quad i \geqq 0,
$$

by

$$
x \rightarrow S q^{2}(x)+x \cdot w_{2}(\xi)
$$


Suppose that $X$ is a closed manifold $M$ of $\operatorname{dim} m$, and let $\xi, \eta$ be two bundles over $M$. Then

$$
\alpha_{\xi}=\alpha_{\eta}: H^{m-2}\left(M ; Z_{2}\right) \rightarrow H^{m}\left(M ; Z_{2}\right)
$$

if, and only if, $w_{2}(\xi)=w_{2}(\eta)$, as may be seen by using Poincaré duality. In particular if we take $\xi$ to be the tangent bundle of $M$, then by Wu [24] $\alpha_{\xi} H^{m-2}\left(M ; Z_{2}\right)=0$, provided $M$ is orientable, and so we have:

LEMMA 5.2. Let $\eta$ be a bundle over a closed orientable m-manifold $M, m \geqq 2$. If $w_{2}(\eta) \neq w_{2}(M)$, then

$$
\alpha_{\eta} H^{m-2}\left(M ; Z_{2}\right)=H^{m}\left(M ; Z_{2}\right) .
$$

Proof of 2.3(c). The first obstruction to $v_{f}$ pulling back to $B O(4 q+1)$ is the class $w_{4 q+2}\left(v_{f}\right)$, which vanishes by hypothesis. The second (and final) obstruction is a coset in $H^{4 q+3}\left(M ; Z_{2}\right)$ of the subgroup $\alpha_{v_{f}} H^{4 q+1}\left(M ; Z_{2}\right)$. (See [9], [10], [20].) Now if $f^{*} w_{2}(N) \neq 0$ then $w_{2}\left(v_{f}\right) \neq w_{2}(M)$, and so by $(5.2), \alpha_{v_{f}} H^{4 q+1}\left(M ; Z_{2}\right)=$ $H^{4 q+3}\left(M ; Z_{2}\right)$, since $M$ is closed. Thus the second obstruction contains zero and hence vanishes, which completes the proof of 2.3(c) in this case.

Suppose on the other hand that

$$
f^{*} w_{2}(N)=0, \quad w_{4 q+1}\left(v_{f}\right) \cdot w_{2}(M)=0 .
$$

Then the theorem follows, as above, by using 5.1 and applying 7.3 of [20]. We omit the details.

Proof of 2.4. We have already done the case $m \equiv 1 \bmod 4$. If $m \equiv 2 \bmod 4$, we use Theorem 1.3 of [21] (applied to the bundle $v_{f}$ ), while if $m \equiv 3 \bmod 4$ we use 5.1 above together with Theorem 1.1 of [21]. We leave the details to the reader.

Proof of 2.5. This follows at once from [21, Theorem 1.3] applied to the bundle $\nu_{f}$.

\section{REFERENCES}

1. A. Borel, Sur la cohomologie des espaces fibrés principaux et des espaces homogènes de groupes de Lie compacts, Ann. of Math. (2) 57 (1953), 115-207.

2. R. Bott, The stable homotopy of the classical groups, Ann. of Math. (2) 70 (1959), 313-337.

3. W. Browder, J. Levine and G. Livesay, Finding a boundary for an open manifold, Amer.

J. Math. 87 (1965), 1017-1028.

4. S. Feder, Immersions and embeddings in complex projective spaces, Topology 4 (1965), 143-158.

5. I. M. James and E. Thomas, Submersions and immersions of manifolds, Inventiones Math. 2 (1967), 171-177.

6. A. Haefliger and M. Hirsch, On the existence and classification of differentiable embeddings, Topology 2 (1963), 129-136.

7. M. Hirsch, Immersion of manifolds, Trans. Amer. Math. Soc. 93 (1959), 242-276.

8. _ On imbedding differentiable manifolds in Euclidean space, Ann. of Math. (2) 73 (1961), 566-571.

9. S. D. Liao, On the theory of obstructions for fiber bundles, Ann. of Math. (2) 60 (1954), 146-191. 
10. M. Mahowald, On obstruction theory in orientable fiber bundles, Trans. Amer. Math. Soc. 110 (1964), 315-349.

11. M. Mahowald and F. Peterson, Secondary cohomology operations on the Thom class, Topology 2 (1964), 367-377.

12. W. Massey, On the Stiefel-Whitney classes of a manifold, Amer. J. Math. 82 (1960), 92-102.

13. - On the Stiefel-Whitney classes of a manifold. II, Proc. Amer. Math. Soc. 13 (1962), 938-942.

14. W. Massey and F. Peterson, On the dual Stiefel-Whitney classes of a manifold, Bol. Soc. Mat. Mexicana (2) 8 (1963), 1-13.

15. J. Milnor, Construction of universal bundles. II, Ann. of Math. (2) 63 (1956), 430-436.

16. G. Paechter, The groups $\pi_{r}\left(V_{n, m}\right)$. I, Quart. J. Math. Oxford Ser. (2) 7 (1956), 249-268.

17. A. Phillips, Submersions of open manifolds, Topology 6 (1967), 171-206.

18. N. Steenrod, The topology of fiber bundles, Princeton Univ. Press, Princeton, N. J., 1951.

19. E. Thomas, Seminar on fiber spaces, Lecture Notes in Math. No. 13, Springer-Verlag, Heidelberg, 1966.

20. - Postnikov invariants and higher order cohomology operations, Ann. of Math. (2) 85 (1967), 184-217.

21. - Real and complex vector fields on manifolds, J. Math. Mech. 16 (1967), 1183-1206.

22. _- Submersions and immersions with codimension one or two, Proc. Amer. Math. Soc. (to appear).

23. H. Whitney, Differentiable manifolds, Ann. of Math. (2) 37 (1936), 645-680.

24. W. Wu, Classes caractéristique et i-carrés d'une variété, C. R. Acad. Sci. Paris 230 (1950), 508-521.

\section{University of CALIFornia, \\ Berkeley, CaLIfornia}

